\title{
Interfacial architecture on the fractal support: polycrystalline gold films as support for self-assembling monolayers
}

\author{
B. Snopok ${ }^{a *}$, P. Strizhak ${ }^{b}$ E. Kostyukevich ${ }^{a}$, V. Serebriy ${ }^{b}$, S. Lysenko ${ }^{a}$, \\ P. Shepeliavii ${ }^{a}$, S. L. Priatkin ${ }^{a}$, S. Kostuykevich ${ }^{a}$, Yu. Shirshov ${ }^{a}$, E. Venger ${ }^{a}$ \\ a Institute of Semiconductor Physics, National Academy of Sciences of Ukraine,pr. Nauki, 45, Kiev, Ukraine, 252650 \\ ${ }^{\mathrm{b}}$ L.V. Pisarzhevskii Institute of Physical Chemistry, National Academy of Sciences of Ukraine, pr. Nauki, 31, Kiev, Ukraine, 252039
}

\begin{abstract}
Multifractal analysis is performed for description of the surface topography of thin polycrystalline gold film. Its structure was modified by annealing at different temperatures in the range $20 \div 200^{\circ} \mathrm{C}$ and films were imaged by Atomic Force Microscopy. Image was analyzed as a collection of layers taken parallel to the mean surface. Fractal subsets with different scaling properties were described by multifractal divergence (e.g. the difference between maximal and minimal values of the $f(a)$ spectrum). This allowed to highlight the effect of the temperature of film annealing on the surface structure. We found that fractal diversity jumps down in the temperature range $130 \div 140{ }^{\circ} \mathrm{C}$. Therefore, phase transition occurs in the system. Below the temperature of the phase transition the surface topography is characterized by high roughness and existence of small-scale irregularities. At critical temperature the surface structure undergoes morphological transition caused by melting of small-scale irregularities. The melting also results in a decrease of the surface roughness due to the flowing down of gold crystallites. A notable feature of the approach is its ability to highlight a possible influence of substrate structure on the adsorption/self-assembling processes at the interface, which may be disturbed by the surface irregularities. The typical and expressive example taken from the self-assembling on the polycrystalline substrate. Particularly, substrate topography determines an order of thiols layers resulting in peculiarities of chemical functionality of obtained material. It was shown that formation of well-ordered monolayers of $\omega$-substituted alkanethiols on gold films occurs only if the freshly evaporated gold films were annealed at temperature more than $c a .120^{\circ} \mathrm{C}$. The analysis of surface peculiarities allows suggesting that this behavior is caused by disappearance of short-scale multifractal structures. Therefore, the multifractal analysis opens a new avenue for both characterization and direct prediction of surface properties. Particularly, it gives a hint regarding a formation of the Euclidean two-dimensional structures at the multifractal substrates.
\end{abstract}

Keywords: polycrystalline gold films, fractal dimension, atomic force microscopy, phase transition, annealing, surface topography, reconstruction, fractal diversity, multi-fractal, self-assembling monolayers, thiols

Paper received 17.09.99; revised manuscript received 18.10.99; accepted for publication 21.10.99.

\section{Introduction}

Specific chemical functionality (i.e. molecular recognition, energy transfer, chemical conversions, etc.) may be only realized within a definitive length scale of structured matter [1]. Particularly, a certain level of functional complexity is a result of combining smaller subunits that are spatially arranged in a specific manner. Regulation of spatial complexity allows one to realize desired functional goal of a material. This rather broad concept encompasses a wide variety

\footnotetext{
* Corresponding author: (e-mail): Snopok@isp.kiev.ua; (phone): (+38044) 26556 26; (fax): (+38044) 2651827.
}

of issues of current technological demand [2]. For instance, sensing optical devices play a central role in optoelectronic intelligent massifs realizing the principal of «chemical imaging» [3]. Further development of these intelligent devices requires an integration of two-dimensional molecular recognition interfaces and transducers transformation of the primarily recognition response into a signal of chemical sensor [4].

Chemical processes at interfaces are governed by three main factors: molecular functionality, distribution of active units, and geometry of the surface [5]. Whereas relative more tremendous knowledge have been accumulated related to the first, and, to a certain extent to the second factor, a little is known regarding a role of surface irregularities for the 


\section{B. Snopok et al.: Interfacial architecture on the fractal support:...}

processes of supramolecular aggregation [6]. There is also no clear understanding of the surface irregularity effect on interfacial functions. To highlight these problems, it is not sufficient either knowledge of molecular properties of a matter in a solution or knowledge of properties of atomically flat surfaces of the same matter. Thus, the expected properties of the resulting assembly can not be readily estimated from the properties of its constituents without considering additional factors stemming from the real substrate structure of physical transducers. The structural peculiarities of surface topography are especially important for promising set of optoelectronic sensors. Among them particular interest is directed towards development of those sensors which are based on evanescent wave phenomena [7], in particular surface plasmon resonance (SPR) [8]. The surface structure of thin film of noble metals (i.e. Au and Ag) plays a crucial role in overall performance of these sensing units. Design of the surface structure allows one to achieve a proper functioning of transduction mechanism as well as to support the formation of two-dimensional layer of receptors. Therefore, further development of these smart devices demands further development of powerful tools for characterization of surface irregularities.

The simplest and the most convenient way for improving the performance of SPR-based sensors is based on lowtemperature treatment of native gold films. The desired result is achieved due to the surface reconstruction [9-10]. However, in spite of relative large experimental materials related to this problem, a little is known about surface transformation processes caused by annealing and their influence the structure and chemical functionality of interfacial architectures thereon. One of the typical and expressive example may be taken from the self-assembling on the polycrystalline substrate. Particularly, substrate topography determines an order of thiols layers resulting in peculiarities of chemical functionality of obtained material. Our experiments have shown that formation of well-ordered monolayers of w-substituted alkanethiols on gold films occurs only if the

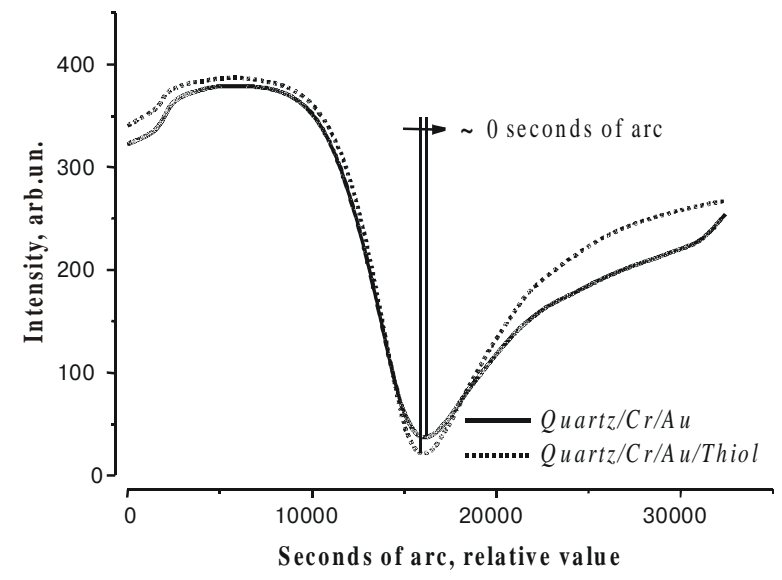

a freshly evaporated gold films were annealed at temperature more than $c a .120^{\circ} \mathrm{C}$. The data presented in Fig. 1 indicated that formation of dodecanthiol monolayer on the freshly evaporated gold surface do not change position of the resonance SPR angle, whereas the contact angle measurements testify to existing hydrophobic organic layer on the surface. This result can be explained by the formation of non-ordered layer. In contrary, SPR investigations of the same process on the surface of annealed gold films at $120^{\circ} \mathrm{C}$ shown that determined SPR shift ( $c a .2050$ seconds of arc) is in good accordance with theoretical value ( $c a .2100$ seconds of arc) calculated in the frame of Fresnel model.

Thus, the task of our investigation was to study the effect of low-temperature annealing onto gold surface with aim to understand how the temperature treatment of gold films change the surface topography and how surface peculiarities influences the formation of self-assembled monolayers of alkanethiols.

\section{Theoretical background}

Three different approaches are commonly used for a characterization of surface irregularities as well as for a description of the topographical peculiarities of thin films. Particularly, these approaches may be based on the analysis of experimental results obtained by profilometer measurements (i.e. AFM imaging) $[11,12]$ or quasi-elastic light scattering [13]. First, scalar approach refers only to the root mean square roughness (rms. roughness) that characterizes a deviation of spatial irregularities from a zero set [15]. The zero set gives the reference plane defined as the plane of zero height, i.e. the sum of surface's heights taken relatively to zero set equals zero. Second, vector approach is usually based on the analysis of quasi-elastic light scattering [16]. Treating the power spectral density (PSD) as a superposition of regular periodic function allows one to describe spatial correlation of surface structure. The third approach is based on the fractal or multifractal analysis of the surface topogra-

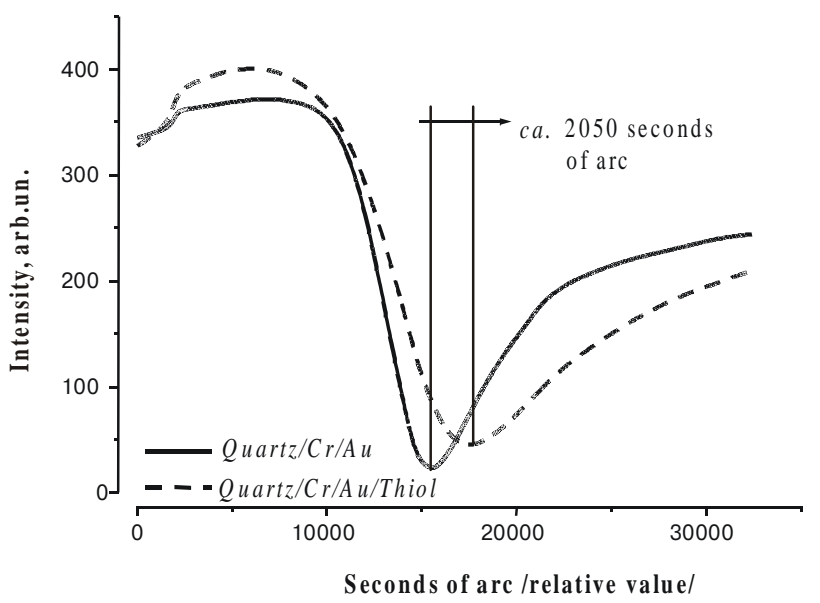

b

Fig. 1. The SPR reflectivity curves for a clean gold surface (solid line) and the same surface after the self-assembling of dodecanthiol (dot line) for untreated freshly evaporated gold films (a) and annealed at $120^{\circ} \mathrm{C}(\mathrm{b})$. The shift in the angle of minimum reflectivity upon the adsorption of material is used to determine the presence of the adsorbed layer. The theoretical value for the shift obtained via complex Fresnel calculations is ca. 2100 seconds of arc. 


\section{B. Snopok et al.: Interfacial architecture on the fractal support:...}

phy [17-18]. It characterizes a surface as a collection of self-similar fractal subsets with different scaling properties.

Historically, scalar (rms. roughness) or one parameter approach is the first and the most common method for the characterization of surface irregularities [14]. It ignores any spatial correlation within the surface. Approaches based on the PSD function or autocorrelation function may be considered as the next level of the description of the surface profile [15]. However, the analysis of real objects using theoretical description of rough surfaces given by PSD function can not be adequately interpreted. It is especially clear if one needs to estimate effective interaction of the surface with substrate [19]. Therefore, it appears a necessity to obtain a prior information about statistical peculiarities of distribution of surface irregularities [16]. Another way of surface characterization can be attributed to fractal or multifractal scaling [20].

Many structures in nature exhibit fractal properties, i.e. some measure of these structures depends on the scale [21]. Like most surfaces, those of thin films are rough when viewed at microscopic level. The closer one looks at the surface the rougher the surface appears. It is caused by the non-regular way that the film particles are packed. This gives the film surface a fractal quality and hence the surface should have a measurable fractal dimension. On this basis various approaches were developed in literature to analyze rough surfaces in terms of fractal dimensions [22]. These approaches are based on an assumption that self-affinity of the rough surface does not depend on the direction, i.e. fractal scaling is the same in all directions. The more deep understanding of self-affinity is based on the analysis of the rough surface properties in $2+1 \mathrm{D}$ case, i.e. if we assume that one type of self-affinity exists in $X Y$ plane and another type exists in Z-direction. Particularly, it was shown that the standard deviation of the surface height scales with the length scale in the $X Y$ plane [23].

We suggest that more informative description may be based on the multifractal analysis of a rough surface if the surface is considered as a collection of layers taken parallel to zero set. Each layer forms 2D structure that can be analyzed in terms of multifractal measures. The surface height $(H)$ is considered as a parameter, i.e. we analyze the multifractal structure of fractal layers at a given value of height $(H=$ const $)$. Properties of the rough surface are characterized by dependencies of multifractal measures on the surface height.

The fractal measures of each layer may be calculated using the box-counting technique [24]. It is based on the portioning the object into equally sized cubes (in our $2 \mathrm{D}$ case - squares) of size $\varepsilon$. The spatial arrangement of points determines $P(m, \varepsilon)$ which gives the probability that there are $\mathrm{m}$ points within a $\mathcal{\varepsilon}$-cube centered around arbitrary point. $P(m, \varepsilon)$ is normalized

$$
\sum_{m=1}^{N} P(m, \varepsilon)=1 \text {. }
$$

Generalized fractal dimensions $\left(D_{q}\right)$ are derived from the Renyi information

$$
I_{q}(\varepsilon)=\frac{1}{1-q} \log \sum_{m=1}^{N} P^{q}(m, \varepsilon)
$$

using the following expression

$$
D_{q}=\lim _{\varepsilon \rightarrow 0} \frac{I_{q}(\varepsilon)}{\log (1 / \varepsilon)} .
$$

This expression indicates that the Renyi information scales with the length scale [25].

Generalized fractal dimensions defined by Eq.(3) have an evident relation to usual fractal measures that are commonly used in an analysis of the fractal properties of materials. $D_{0}$ gives fractal dimension of the support of the measure, $D_{1}$ is the information dimension, and $D_{2}$ gives the correlation dimension. It has been emphasized that for multifractal objects the probability scales as the exponent $\alpha$ whose value depends on the location of the cube and can be taken on a finite range of values. The singularities corresponding to a given value of $\alpha$ are distributed over a subset of Hausdorff dimensions $f(\alpha)$. The function $f(\alpha)$ is called the multifractal spectrum. It is defined via the following Legendre transformation of $D_{q}$ [24-25]:

$$
\begin{aligned}
& \alpha(q)=\frac{d}{d q}\left[(q-1) D_{q}\right] \\
& f(\alpha)=q \alpha+(1-q) D_{q}
\end{aligned}
$$

The $f(\alpha)$ spectrum has well-known interpretation in terms of the distribution $\left\{P_{m}\right\}=\{P(m, \varepsilon \rightarrow 0)$. For any particular $P_{m}$ a corresponding $\alpha_{m}$ is defined by $P_{m}=\left(a / r_{g}\right)^{\alpha m}$, where $a$ is the particle diameter, and $r_{g}$ is the cluster radius of gyration. Then the total number of particles with $P_{m}$ in a range $d \alpha, N(\alpha) d \alpha$, defines a quantity $f(\alpha)$ via $N(\alpha)=\left(a / r_{g}\right)^{f(\alpha)}$, and $f(\alpha)$ is independent of $r_{g}$ for multifractals. The $f(\alpha)$ spectrum gives the quantitative measure for multifractal object that consists of a number of subsets with different fractal dimensions.

The values of $f(\alpha)$ and $\alpha$ at a particular value of $q$ satisfy the tangent condition

$$
\frac{d f(\alpha)}{d \alpha}=q \text {. }
$$

By definition, $f(\alpha)$ is an intrinsically positive quantity, since $N(\alpha) \geq 1$. In general, $f(\alpha)$ is a nonnegative, convex function defined in an interval $0<\alpha_{\min } \leq \alpha \leq \alpha_{\max }<\infty$. The $f(\alpha)$ spectrum has a maximum at $\alpha_{0}$ the value of which is determimed by the condition $q=0$. The maximum equals to the dimension of the support, i.e. the fractal dimension of the subset of points where the investigated quantity is not zero. The $f(\alpha)$ curve lies below the bisector $f(\alpha)=\alpha$ except the single point at $\alpha(q=1)$ where both curves touch.

\section{Experimental Section}

The sample support was a quartz plate of dimensions $41 \times 19 \times 1.5 \mathrm{~mm}^{3}$ with the area rms. roughness $c a .1 .1 \mathrm{~nm}$. For the sample formation, the upper side of the support plate was covered with a $c a .5 \mathrm{~nm}$ adhesive layer of $\mathrm{Cr}$, followed 


\section{B. Snopok et al.: Interfacial architecture on the fractal support:...}

by $45 \mathrm{~nm}$ of $\mathrm{Au}$. During the deposition the plate was supported at $22{ }^{\circ} \mathrm{C}$ with subsequent holding (three hour) at high vacuum $\left(5 \cdot 10^{-4} \mathrm{~Pa}\right)$ before removing from the evaporation equipment (VUP-5M). Annealing of films was conducted in air atmosphere during $40 \mathrm{~min}$ at different temperatures $\left(20,80,120,150\right.$ and $\left.200^{\circ} \mathrm{C}\right)$. Samples were used for measurements without any additional cleaning procedures prior to experiment.

We used tapping-mode under AFM imaging because our special interest was focused on the greatest possible set of irregularities of surface relief involving both tops and valleys of the surface. The AFM imaging was undertaken using a commercial Nanoscope IIIa (Digital Instrument, Santa-Barbara, CA) equipped with an $80-\mu \mathrm{m}$ scanner. Scans were performed in the tapping force mode using commercially available AFM tips (silicone nitride). Scanning frequency was approximately $1 \mathrm{~Hz}$ in all experiments.

Images were stored as $256 \times 256$ matrixes. These matrixes were analyzed using software which source code was written on $\mathrm{C}++$. The range of box sizes used to calculate the Renyi information was from 4 to 40 pixels that corresponds to linear size in the range of $15-200 \mathrm{~nm}$. We emphasize that in all calculations we analyze the fractal measures only for layers containing more than 10000 pixels.

\section{Results and discussion}

Our initial discussion focuses on the AFM characterization of the gold thin films annealed at different temperatures. We perform an attempt to describe the peculiarities of the surface topography using conventional manner of characterization, i.e. using scalar and vector approaches. Then we present a detailed analysis of these surfaces using multifractal approach, in particular, using the multifractal scaling of layers which are parallel to zero set. The final subsection is devoted to the discussion of multifractal approach for analysis and characterization of surface topography. We also perform an attempt to highlight physical mechanisms that governs surface transformation under annealing.

The topography of untreated and annealed gold coated plates. The gold-coated plates were initially imaged by AFM. The AFM image shown in Fig. 2 (bottom) illustrates that the surface is not atomically smooth. The grain diameter of the gold coating is in the range from 20 to $30 \mathrm{~nm}$. The surface roughness exhibits a maximum height variation of $2.5 \mathrm{~nm}$ for a $1000 \mathrm{~nm}$ scan, with the rms. roughness ca. $0.93 \mathrm{~nm}$. Annealing of the films leads to the nonmonotonic change of the roughness. The rms. roughness initially grows to $\mathrm{ca} .1 .1 \mathrm{~nm}$ at $80^{\circ} \mathrm{C}$, then it reduces to $0.84 \mathrm{~nm}$ at $120{ }^{\circ} \mathrm{C}$, increase up to $0.95 \mathrm{~nm}$ at $150{ }^{\circ} \mathrm{C}$ and decreases to $0.68 \mathrm{~nm}$ at $200{ }^{\circ} \mathrm{C}$. This result is in agreement with literature data that emphasize irregular changes of surface roughness under low-temperature annealing of thin polycrystalline films [26]. However, surface roughness, which is a single characteristic of the surface, can not be simply used for direct and definitive characterization of the reconstruction process of the surface.

The AFM images of films shown in Fig. 2 resolve features which are consistent with local surface structure at SQO, 2(3), 1999
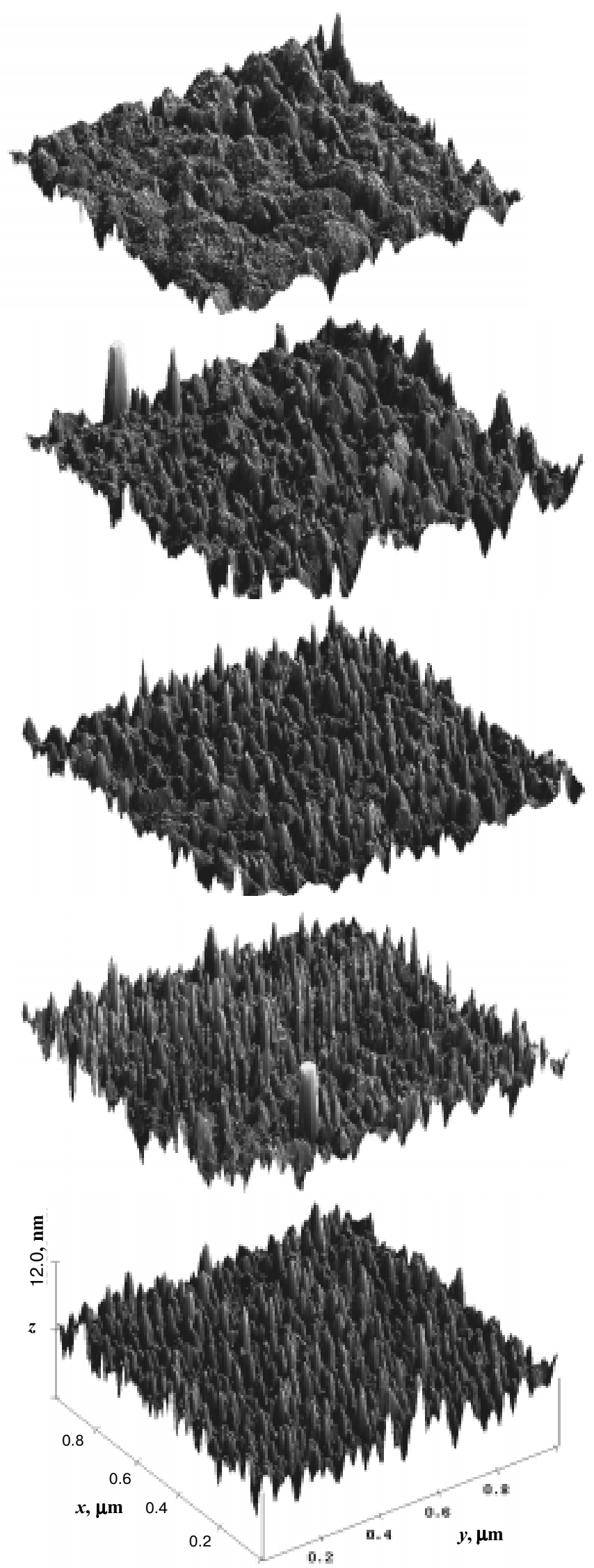

Fig. 2. Atomic force microscope images (1000 nm square scan) of the untreated gold surface $\left(20^{\circ} \mathrm{C}\right.$, bottom $)$ and AFM images of treated at different temperatures in air (from bottom to top): $20,80,120,150$ and $200{ }^{\circ} \mathrm{C}$. 


\section{B. Snopok et al.: Interfacial architecture on the fractal support:...}

nanometer scale. This follows from the PSD functions presented in Fig. 3. These PSD functions are obtained for the AFM images shown in Fig. 2. The PSD functions give statistical characteristics of the object indicating that certain substructures of given dimensions are presented at the surface. The data shown in Fig. 3 allow us to conclude that all samples are characterized by similar statistical properties, i.e. statistics of different objects on the surface does not depend on the annealing temperature. There are three specific regions inherent for all PSD curves, namely, below $10 \mathrm{~nm}$, in the range from 10 to $60 \mathrm{~nm}$ and above 60 $\mathrm{nm}$. In the first region all curves show the same dependence via surface wavelength and, possibly, represent the statistical properties of small relief height inside areas around peaks and bottoms of crystallites. The next range of surface wavelengths characterizes the peculiarities of height distribution in the range of lateral waveform specific to the dimension of separate crystallites and cavities. It is necessary to stress that essential changes in PSD spectrum are observed in this region. They take place only at temperatures above $150{ }^{\circ} \mathrm{C}$. Therefore, the grain diameter of dominant crystallites does not essentially change at low temperatures. Moreover, PSD spectrum indicates that certain surface objects with specific surface wavelength $c a .20 \mathrm{~nm}$ disappear at high temperatures. Finally, we may conclude that the surface structure is more organized for thin films annealed at temperatures above $150{ }^{\circ} \mathrm{C}$. The intersection point of PSD functions above $60 \mathrm{~nm}$ bound the region that represents the statistical distribution of groups of crystallites at the surface. Statistical properties of films annealed at different temperatures may be separated into two groups. The films annealed at 20,120 and $200{ }^{\circ} \mathrm{C}$ belong to the first group, and those films annealed at 80 and $150^{\circ} \mathrm{C}$ belong to the second one. The second group is characterized by more high values of PSD functions which is in agreement with relation between rms. roughness and the annealing temperature.

The discussion of surface properties of thin gold films

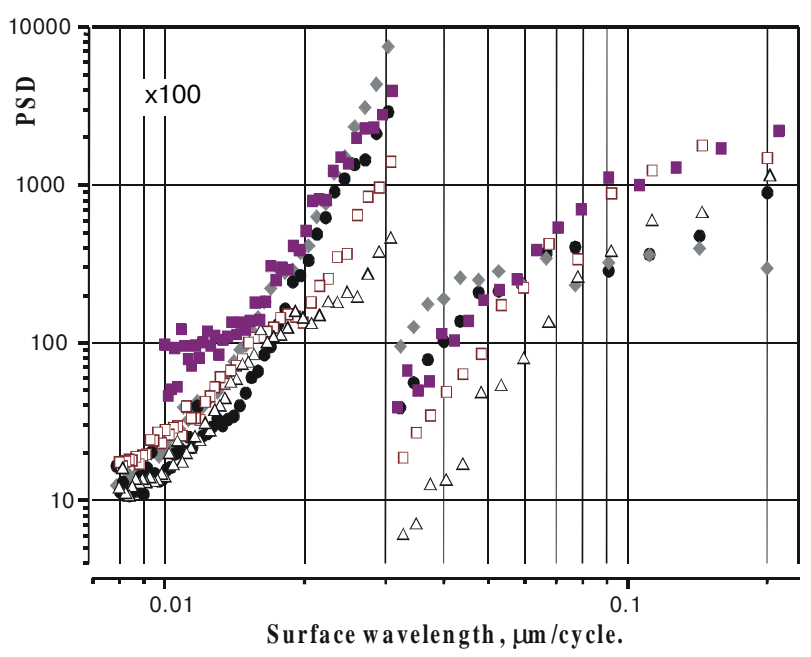

Fig. 3. Two - dimensional isotropic Power Spectral Densities functions for untreated gold surface ( $\diamond, T=20^{\circ} \mathrm{C}$ ) and annealed gold films at different temperatures: $80^{\circ} \mathrm{C}(\bullet), 120^{\circ} \mathrm{C}(\bullet), 150^{\circ} \mathrm{C}(\square)$ and $200^{\circ} \mathrm{C}(\Delta)$. annealed at different temperatures permits us to conclude that the analysis based on either statistical distribution of heights (scalar approach) or PSD functions (vector approach) does not allow one to describe physical mechanism of surface formation or peculiarities of rough surfaces of polycrystalline films. The main reason of this is a loss of information about local structures caused by statistical averaging. Either scalar or vector approaches do not take into account a dependence of properties of surface structures on the surface height.

To analyze height distribution of gold surface we calculated probability distribution functions for the height of $\mathrm{Au}$ relief relative to zero set. Fig. 4 gives these functions for annealed films in comparison with untreated sample. All probability distribution functions are characterized by bellshaped form. They are centered at the vicinity of zero set. Annealing at $80{ }^{\circ} \mathrm{C}$ results only in redistribution of probability at $H<0$, i.e. below zero set. Namely, a probability of irregularities that exist at low values of $H<-1.2 \mathrm{~nm}$ increases for the film annealed at $80{ }^{\circ} \mathrm{C}$ comparing with the untreated sample. The opposite effect is observed for the probability in the range $-1.2 \mathrm{~nm}<H<0 \mathrm{~nm}$. This indicates that valleys of the rough surface become smoother. This is caused by flowing down of gold particles that were primarily located at the tops of short-scale irregularities inside these valleys. The right side of the probability distribution function is identical for both samples indicating that there are no changes for the fractures located at the tops of crystallites.

Shown in Fig. 4b is the comparison of the distribution functions of untreated and annealed at $120{ }^{\circ} \mathrm{C}$ samples. It illustrates that the transformation of the surface results in an increase of the probability around zero set $(H=0 \pm 0.5 \mathrm{~nm})$. Simultaneously, the probability decreases at low and high values of $H$. Therefore, all crystallites, including those located around «tops» and «valleys», undergo short-scale smoothing. Since the shape of distribution curve does not change essentially under this reconstruction, we may assume that the long-range profile of the surface remains unaltered.

In the same manner Fig. $4 \mathrm{c}$ gives the comparison of the probability distribution functions for the sample annealed at $150{ }^{\circ} \mathrm{C}$ and untreated sample. It is worth noting that probability distribution function for the sample annealed at $150{ }^{\circ} \mathrm{C}$ does not change essentially comparing to that for the sample annealed at $120{ }^{\circ} \mathrm{C}$. However, two fine features may be distinguished if we compare the data presented in Fig. $4 \mathrm{~b}$ and c. Firstly, at $150{ }^{\circ} \mathrm{C}$ small changes appear near valleys. Secondly, the probability becomes to grow near zero set. Therefore, we may assume that a new topographical structure becomes to form at $T=150{ }^{\circ} \mathrm{C}$. Further increase of annealing temperature up to $200{ }^{\circ} \mathrm{C}$ results in essential narrowing of distribution function as shown in Fig. 4d. All fractures undergo the reconstruction process, and the topology of the total interfacial structure changes.

Layer analysis of gold surface. More deep analysis of rough surface may be based on calculation of fractal dimensions. In this subsection we perform an analysis of multifractal structures of layers chosen at a given value of surface height $(H=$ const $)$. Layers are taken parallely to zero set $(H=0)$. 


\section{B. Snopok et al.: Interfacial architecture on the fractal support:...}

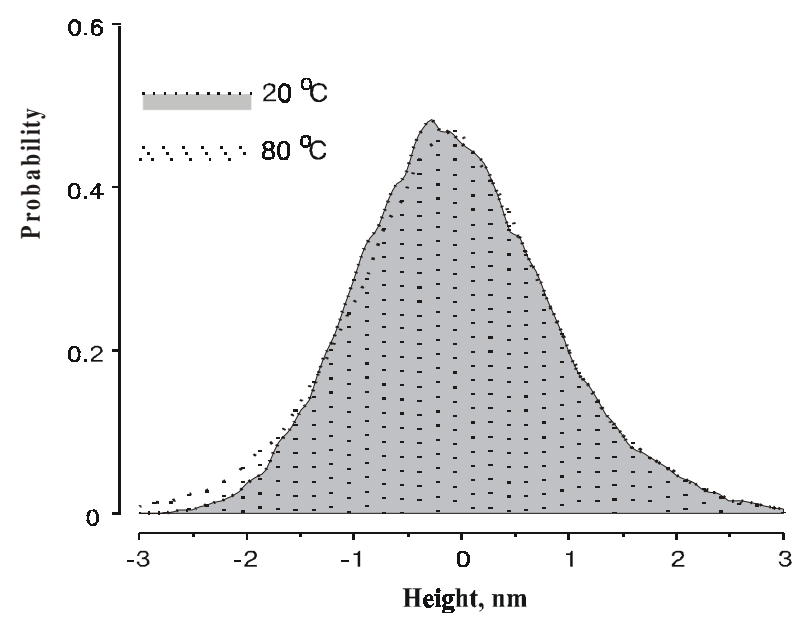

a

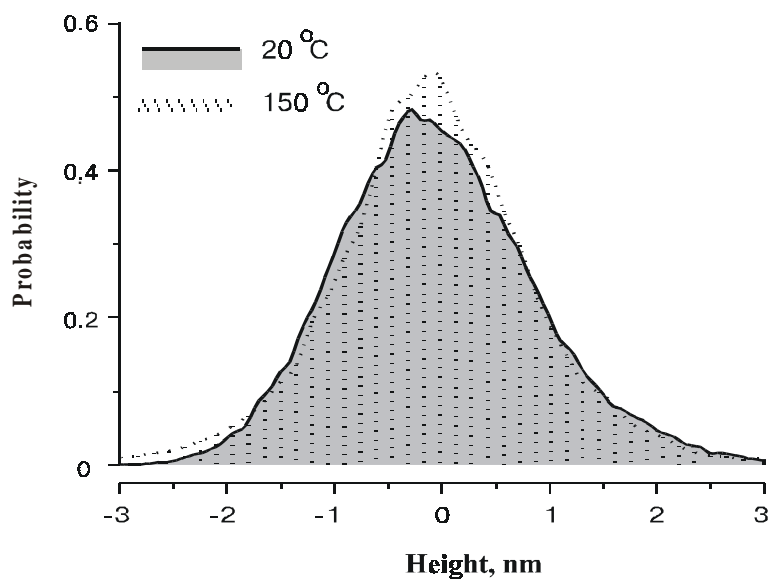

C

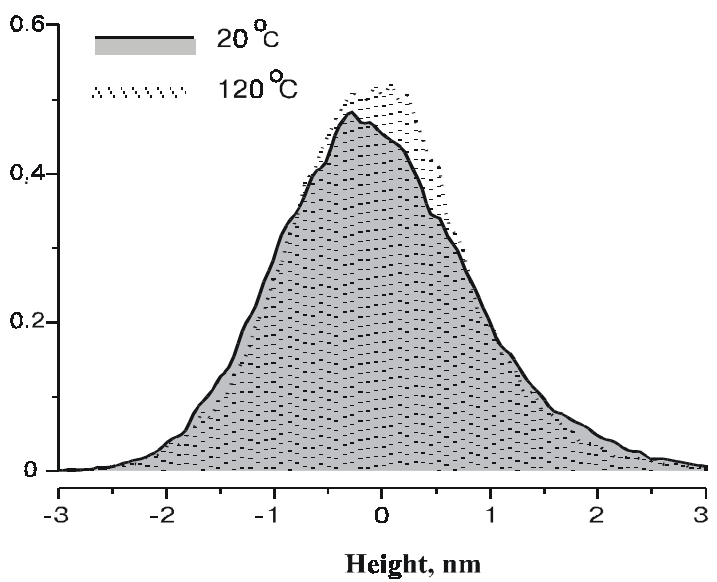

b

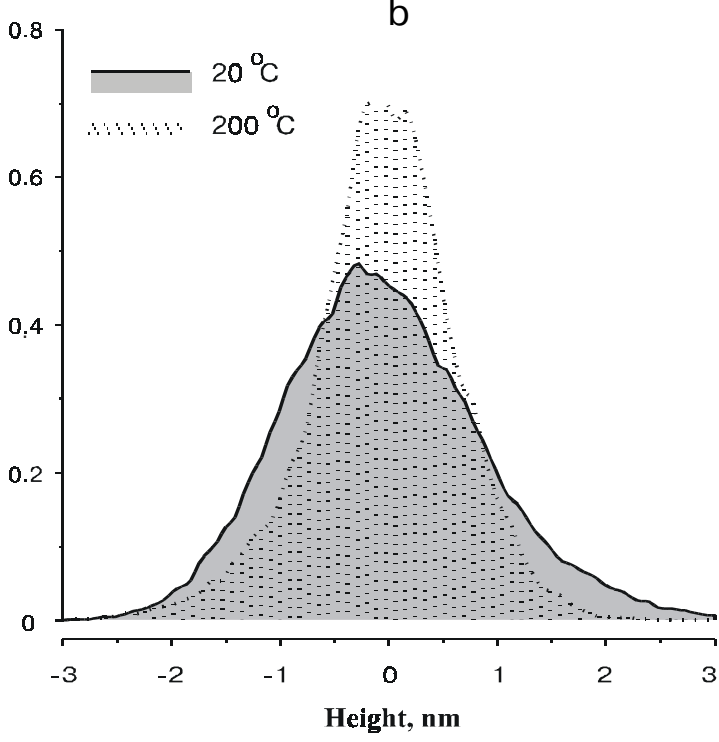

d

Fig. 4. The probability distribution functions of heights for annealed gold films in comparison with untreated sample (from left to right): $80 / 20{ }^{\circ} \mathrm{C}$, $120 / 20{ }^{\circ} \mathrm{C}, 150 / 20{ }^{\circ} \mathrm{C}, 200 / 20{ }^{\circ} \mathrm{C}$.

This approach allows us to describe multifractal properties of the rough surface considering it as a collection of these layers taken in $X Y$ plane. The layer's height plays a role of a parameter (Z-direction).

Fig.5 shows the Renyi information as a function of $\log (L)$ for various positive $\mathrm{q}$ values where the value of $L$ gives the linear scale in $X Y$ plane. Each panel presents the corresponding values of the Renyi information. They were calculated for different five layers of the Au surface obtained at $T=20^{\circ} \mathrm{C}$. The data presented in Fig. 5 indicate that at a given value of $q$ the Renyi information decreases if height of the layer increases. Therefore, the Renyi information monotonically decreases with a growth of the surface width. The dependencies of $I_{q}$ on $\log (L)$ are monotonic for any value of $q$ or $H$. However they are not linear. Their divergence from linear fits becomes more pronounced with either increasing $q$ or increasing $H$. Nonlinear character of these dependencies indicates that each layer has a multifractal structure. Therefore, each layer consists of a set of sub- structures with different fractal measures as shown in Fig.6 which gives dependencies of fractal dimension $\left(D_{0}\right)$, information dimension $\left(D_{1}\right)$, correlation dimension $\left(D_{2}\right)$, and $D_{10}$ on the layer height for Au surfaces annealed at different temperatures. At given values of temperature and $q$ the corresponding fractal dimension decreases with growing $H$. These dependencies become less smooth at high values of $q$. This is caused only by a limited number of points taken into account for calculation of fractal measures.

The data presented in Fig. 7 indicate that the dependence of $D_{q}$ on the layer height is characterized by $S$-shaped function. The value of $D_{q}$ tends to 2 for layers that lies deeper, and $D_{q}$ tends to 0 otherwise. It is worth noting that for nonfractal structure the dependence of $D_{q}$ on $H$ becomes a stepwise function. A tendency of the function $D_{q}(H)$ towards the stepwise function illustrates that fractal structure becomes more ordered and less fractal. Therefore, an increase of annealing temperature increases an order of the layer's structures. It also shifts down the layers with a given 
B. Snopok et al.: Interfacial architecture on the fractal support:...
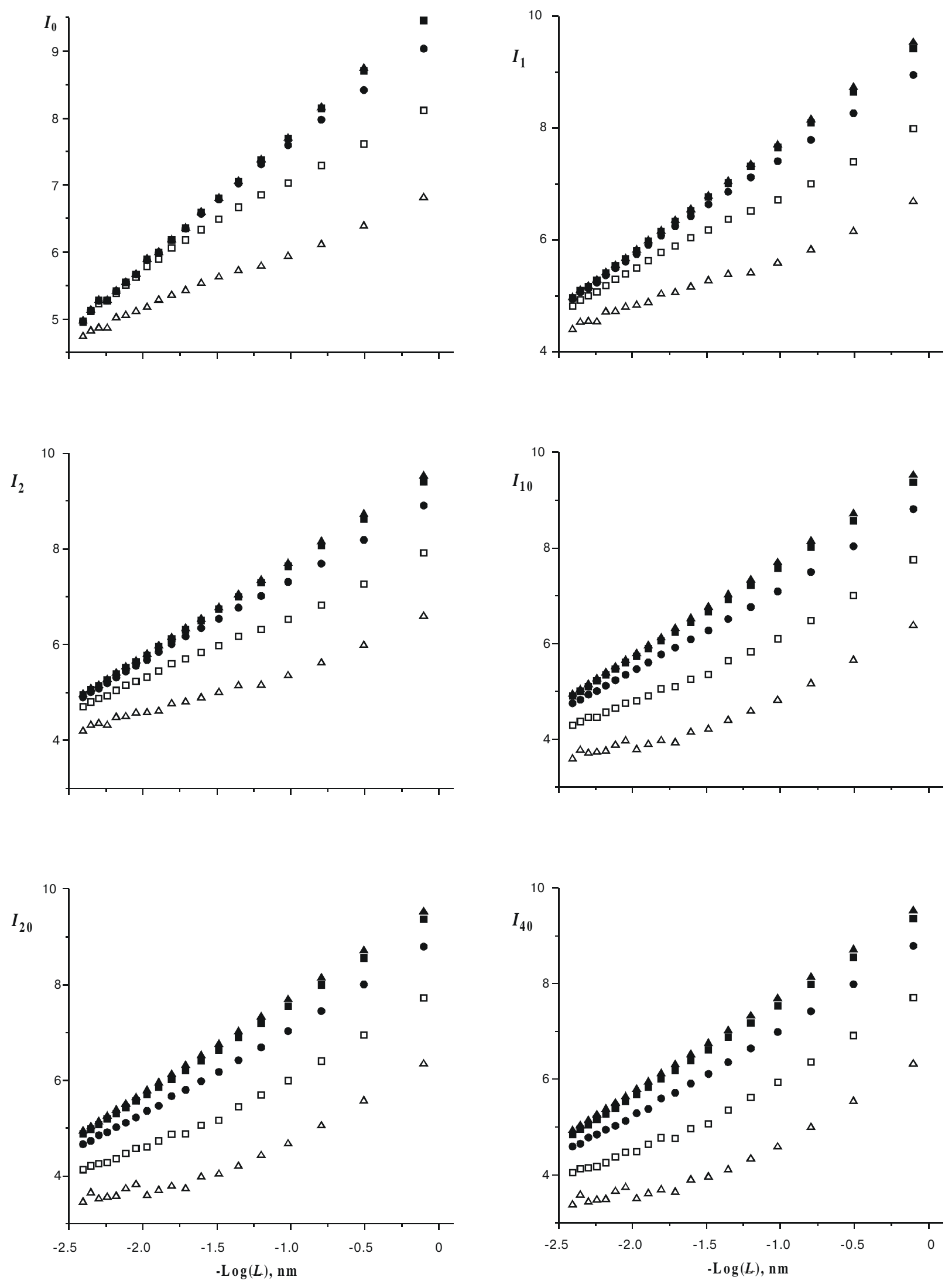

Fig. 5. Dependencies of the Renyi information on the $\log (L)$ at different values of $q\left(I_{0}, I_{1}, I_{2}, I_{10}, I_{20}, I_{40}\right)$ and $H\left(T=20^{\circ} \mathrm{C}, \Delta-1000\right.$, $\mathrm{\square}-5000$, •- $0 \mathrm{~nm},--5000 \mathrm{~nm}, \mathbf{\Delta}--1000 \mathrm{~nm})$. 


\section{B. Snopok et al.: Interfacial architecture on the fractal support:...}
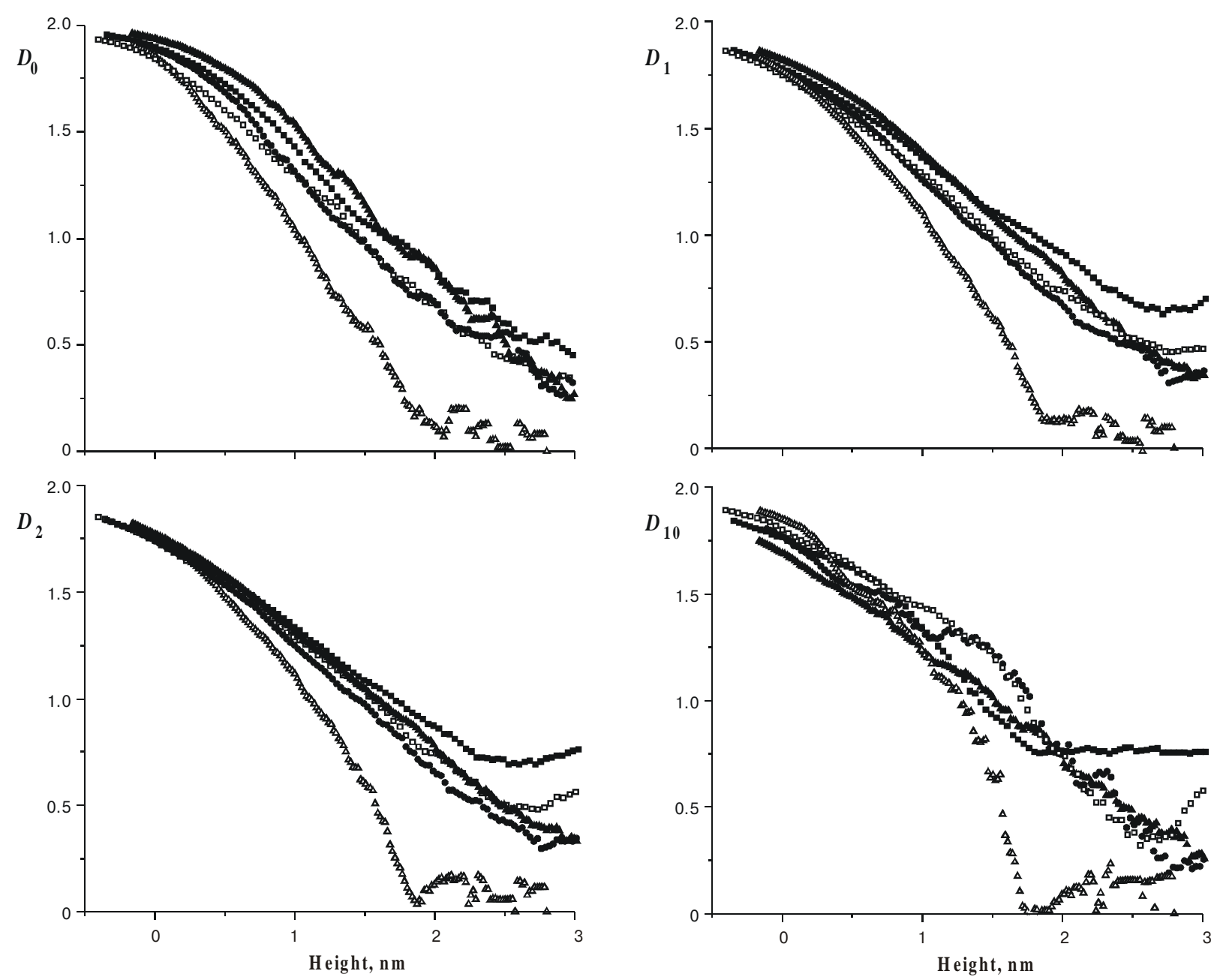

Fig. 6. Dependencies of the values of $\mathrm{D}_{0}, \mathrm{D}_{2}, \mathrm{D}_{1}$ and $\mathrm{D}_{10}$ versus height for different temperatures $\left(\Delta-20{ }^{\circ} \mathrm{C},-80^{\circ} \mathrm{C}, \bullet 120^{\circ} \mathrm{C}\right.$, ㅁ $\left.-150{ }^{\circ} \mathrm{C}, \Delta-200^{\circ} \mathrm{C}\right)$.

value of the fractal dimension as illustrated in Fig. 7. Two top panels in this figure give the temperature dependencies of layer heights with given values of $D_{0}$ and $D_{2}$. Two bottom panels illustrate the temperature effect on these fractal measures for layers located at given heights.

Multifractal formalism. The next step of our analysis is based on calculating the $f(\alpha)$ spectrum according to Eq.(4). The $f(\alpha)$ spectra are shown in Fig. 8a, b for two layers located at $H=0 \mathrm{~nm}$ and $H=1 \mathrm{~nm}$ respectively. Both panels illustrate that $f(\alpha)$ spectrum is represented by monotonically increasing function for any temperature. In almost all cases an increase of annealing temperature shifts the $f(\alpha)$ spectrum up and to the left. The $f(\alpha)$ spectrum saturates at low values of $\alpha$ and may be characterized by $\Delta f=f_{\max }-f_{\min }$, where $f_{\max }$ and $f_{\min }$ are maximal and minimal values of $f(\alpha)$ for the whole range of $\alpha$. The value $\Delta f$ is called fractal diversity. Fig. 9 gives dependencies of $f_{\max }, f_{\min }$, and $\Delta f$ on layer height for the Au surfaces annealed at different temperatures. Left panels illustrate that $f_{\max }$ always decreases with increasing $H$, whereas dependencies of $f_{\min }$ on $H$ are non-monotonic for any annealing temperature. At $T=20^{\circ} \mathrm{C} f_{\min }(H)$ has a minimum located near zero set $(H=0)$. Increasing anneal- ing temperature shifts this minimum to the left at $T=80^{\circ} \mathrm{C}$. The minimum splits into two minima at $T=120^{\circ} \mathrm{C}$. At $T=$ $150{ }^{\circ} \mathrm{C}$ and $T=200{ }^{\circ} \mathrm{C}$ these local minima become less expressed. This behavior of $f_{\max }$ and $f_{\min }$ results in existence of single global maximum for the dependence of $\Delta f$ on $H$ at $T=20^{\circ} \mathrm{C}$, and two global maxima for other temperatures. These two maxima are more pronounced at $T=80^{\circ} \mathrm{C}$ and $T=120^{\circ} \mathrm{C}$.

The maximal value of $\Delta f, \Delta f_{\max }$, which we define as the maximal fractal diversity, always take values near 1.5 for samples annealed at $T<130^{\circ} \mathrm{C}$. The maximal fractal diversity is always less then 0.7 for samples annealed at higher temperatures. Fig. 10a gives the dependence of the maximal fractal diversity on the annealing temperature. These data illustrate that fractal diversity jumps from high to low values in the temperature range $130 \div 140{ }^{\circ} \mathrm{C}$. Therefore, in this temperature range a phase transition occurs in the system. The structure of the Au surface becomes suddenly less diverge in terms of multifractal measures. Fig. 10b shows the dependence of the layer height, at which the multifractal divergence is maximal, on the temperature. This dependence indicates that an increase of annealing temperature 
B. Snopok et al.: Interfacial architecture on the fractal support:...
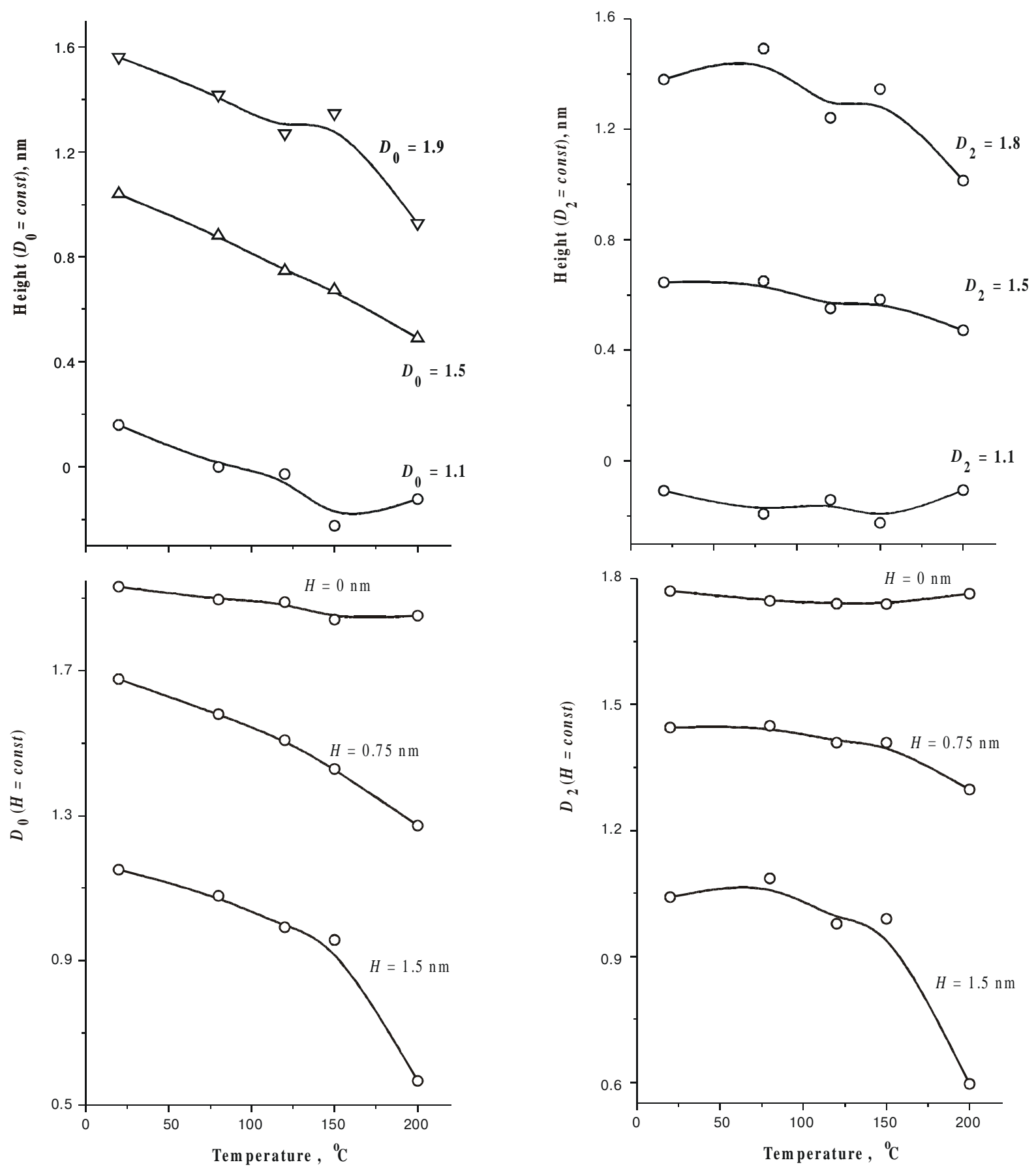

Fig. 7. Temperature dependencies of the layers heights with a given values of $D_{0}$ and $D_{2}$ and temperature dependencies of the $D_{0}$ and $D_{2}$ for the layers with a given distance from zero set.

shifts up the layers with maximal multifractal divergence.

Model of interfacial melting at the surface of polycrystalline gold films. In this subsection we present speculations regarding possible physical mechanism of the annealing treatment of the rough surface. Analysis of surface topography based on scalar, vector and fractal approaches presented in previous subsections permits us to make the following conclusions:

a) at any temperature the portion of small-scale surface irregularities decreases from deep layers to lay- ers located at high $H$, i.e. the shape of crystallite's peaks is more smooth comparing with the shape of crystallite's valleys;

b) at $T<130 \div 140{ }^{\circ} \mathrm{C}$ the crystallites profile does not depend essentially on the annealing temperature;

c) at critical temperature $T=130 \div 140{ }^{\circ} \mathrm{C}$ the phase transition occurs:

i) below critical temperature the surface topography is characterized by high roughness and existence of small-scale irregularities;

ii) at critical temperature the surface undergoes 
B. Snopok et al.: Interfacial architecture on the fractal support:...
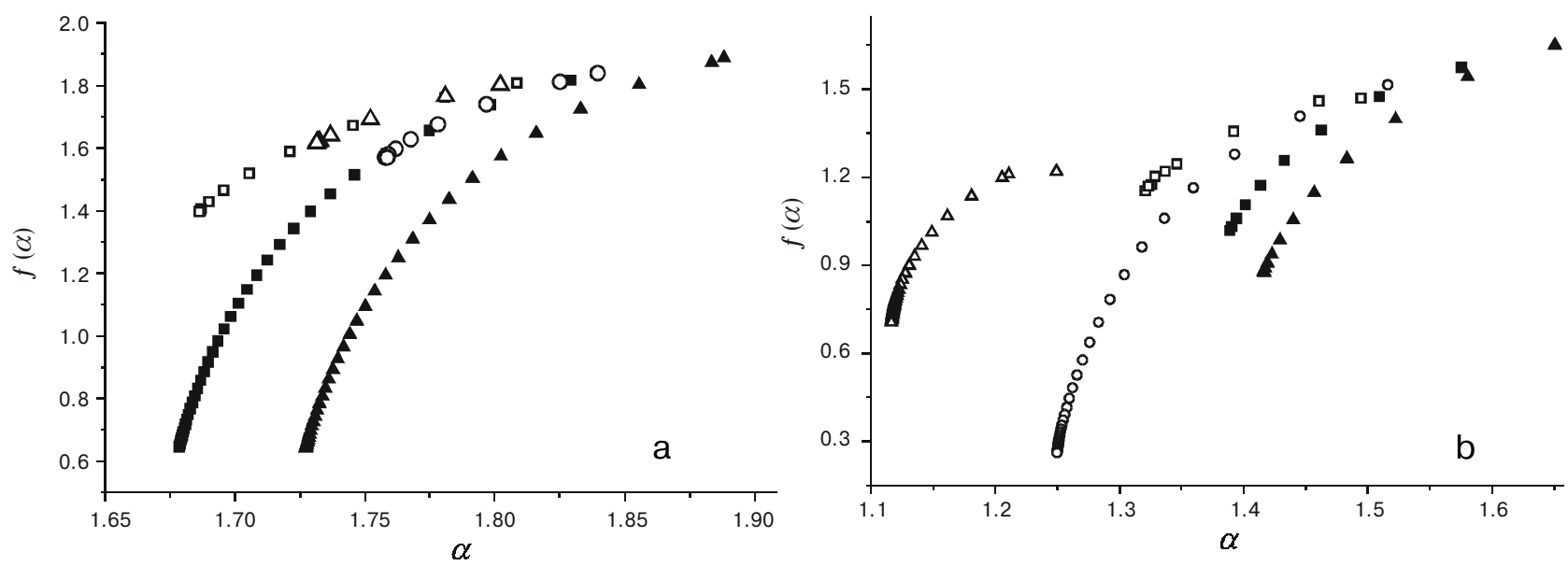

Fig. 8. The $f(a)$ spectrum at two heights $(\mathrm{a}-H=0 \mathrm{~nm}, \mathrm{~b}-H=1 \mathrm{~nm})$ for different temperatures $\left(\boldsymbol{\Delta}-20{ }^{\circ} \mathrm{C}, \boldsymbol{\square}-80{ }^{\circ} \mathrm{C}, \bullet-120{ }^{\circ} \mathrm{C}\right.$, ㅁ $\left.-150^{\circ} \mathrm{C}, \Delta-200^{\circ} \mathrm{C}\right)$.
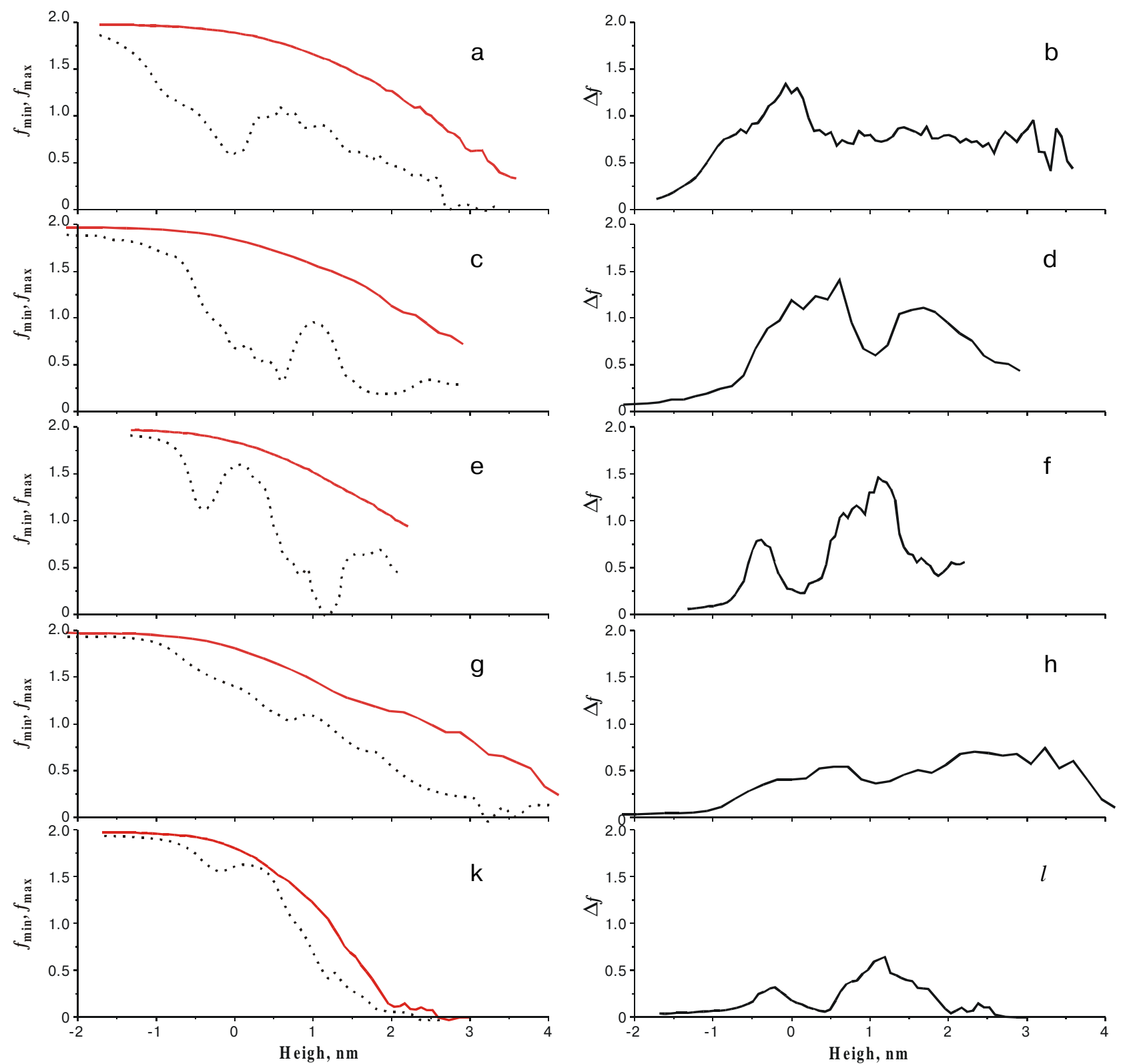

Fig. 9. Dependencies of $f_{\max }, f_{\min }$ (left panels) and $\Delta f$ (right panels) on the layers height at different temperatures (a, b - $20{ }^{\circ} \mathrm{C}, \mathrm{c}, \mathrm{d}-80{ }^{\circ} \mathrm{C}$, $\left.\mathrm{e}, \mathrm{f}-120^{\circ} \mathrm{C}, \mathrm{g}, \mathrm{h}-150^{\circ} \mathrm{C}, \mathrm{k} . l-200^{\circ} \mathrm{C}\right)$. 


\section{B. Snopok et al.: Interfacial architecture on the fractal support:...}

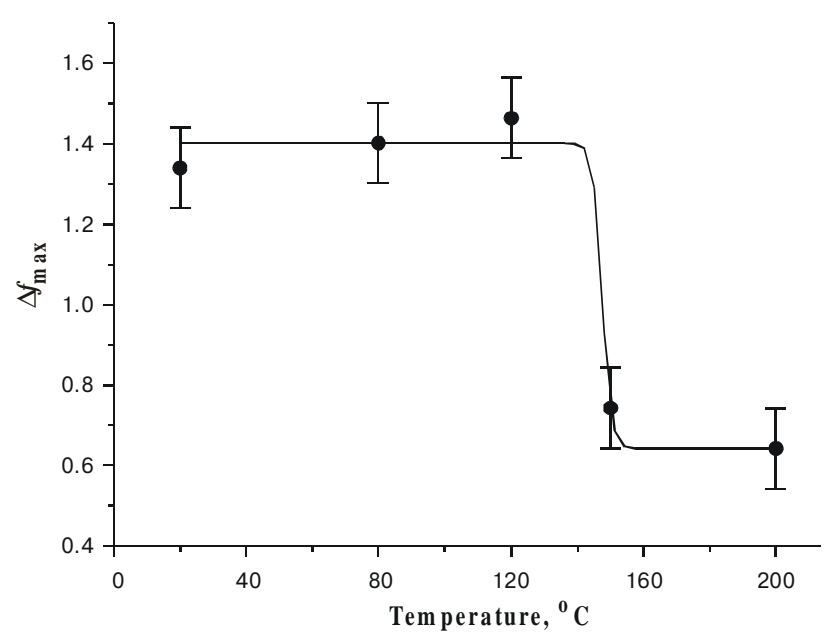

a

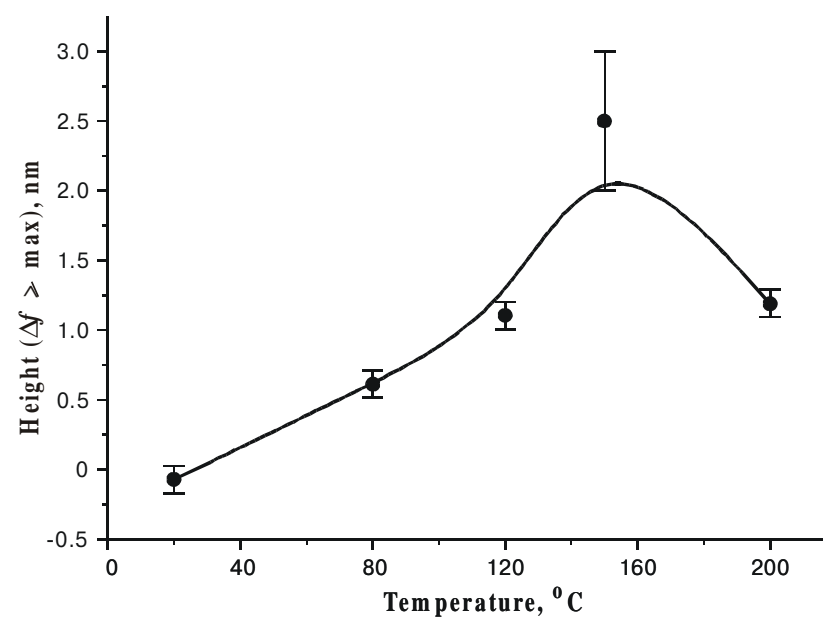

b

Fig. 10. Temperature dependencies of $\Delta f$ (a) and heights of layers with maximal fractal divergence $\Delta f(\mathrm{~b})$.

morphological transition caused by melting of all irregularities;

iii) above critical temperature the surface structure becomes more ordered and less fractal;

d) transverse spectral distribution of multifractal divergence «moves» to upper layers of the Au surface if annealing temperature increases;

e) an increase of annealing temperature causes elimination of the surface fractures with high values of $f_{\max }$

f) portion of fractures with a given value of $f_{\min }$ does not depend monotonically on the annealing temperature.

These results allow us to suggest the following mechanism of the annealing treatment of rough gold surface. In the temperature range $20-80{ }^{\circ} \mathrm{C}$ the surface is characterized by melting of small-range irregularities which are located at the bottom of surface cavities. It results in an increase of the mean surface roughness and the PSD values. Reconstruction of small-range fractures takes place at the
$T=80-130{ }^{\circ} \mathrm{C}$ for all crystallites. The shape of surface relief from «rough» to «flat» changes only on the small scale in $X Y$ plane. Whereas, the long-scale fractures still survive. We assume that at $T$ approx. $130^{\circ} \mathrm{C}$ all small-scale irregular fractures disappear, and the surface of each crystallite become smooth. At this temperature the reconstruction of surface's valleys is finished. Their structures do not change essentially at higher temperatures. An increase of annealing temperature up to $150{ }^{\circ} \mathrm{C}$ initiates a transformation of the tops of crystallites. It results in smoothing top regions of crystallites. For a given crystallite the temperature of this process depends on the surface tension and its curvature. Further increase of temperature leads to formation of quasiflat area.

\section{Concluding remarks}

Controlling the surface topography of physical transducers is a major concept for a proper design of bio- and chemosensitive device. Our study show that multifractal formalism gives a suitable way to characterize the rough surface of polycrystalline gold films more specifically than other approaches. Namely, a dependence of multifractal characteristics on annealing temperature allowed us to describe the gold surface transformations that take place at low-temperature annealing of gold films. A notable feature of the approach is its ability to highlight a possible influence of substrate structure on the adsorption/self-assembling processes at the interface, which may be disturbed by the surface irregularities. The typical and expressive example taken from the self-assembling on the polycrystalline substrate. Particularly, substrate topography determines an order of thiols layers resulting in peculiarities of chemical functionality of obtained material $[27,28]$. Our experiments have shown that formation of well-ordered monolayers of $\omega$-substituted alkanethiols on gold films occurs only if the freshly evaporated gold films were annealed at temperature more than ca. $110^{\circ} \mathrm{C}$. The results presented in previous section allow us to suggest that this behavior is caused by disappearance of short-scale multifractal structures. Therefore, the multifractal analysis opens a new avenue for both characterization and direct prediction of surface's properties. Particularly, it gives a hint regarding a formation of the Euclidean two-dimensional structures at the multifractal substrates. Taking in consideration that thin gold film is the key element of surface plasmon resonance transducer [29], we may conclude that changing multifractal structure of gold film by appropriate treatment affects the efficiency of transduction mechanism. For example, the analysis of excitation conditions of surface plasmons in the films annealed at different temperatures indicates that optimal response may be achieved for films annealed at $120^{\circ} \mathrm{C}$. Therefore, the multifractal structure of long-range fractures plays an important role for the inhomogeneously grating and governs the surface field enhancements of localized surface plasmon [30].

Comparison of multifractal scaling of the rough gold films with their properties gives a useful tool to characterize and even to predict properties of a given material. Moreo- 


\section{B. Snopok et al.: Interfacial architecture on the fractal support:...}

ver, our analysis reveals those properties of the surface, which are controlled by appropriate treatment. This opens a way to a proper design of a surface for a construction of a desirable transducer. Multifractal analysis of surface layers taken parallel to zero set permits not only to visualize the surface reconstructing processes (e.g. phase transition). It also allows one to understand driving forces of surface transformations and gives physical reasons for the effect of this transformation on the properties of interfacial structures. Particularly, our analysis of multifractal scaling of rough gold films revealed the mechanism of the low-temperature annealing that can be used for direct optimization of rough surfaces in relation to required properties.

\section{References}

1. G. Decher «Layered Nanoarchitectures via Directed Assembly of Anionic and Cationic Molecules» in Comperhensive Supramolecular Chemistry, Ed. G.Abberti and T. Bein, Elsevier Science Ltd., 1996.

2. E.Delamarche, B.Michel, H.A.Biebuyck, C.Gerber «Golden Interfaces: The Surface of Self-Assembled Monolayers», Advanced Materials, 8, pp.719-729, 1996.

3. U.Weimar, W.Gopel «Chemical Imaging: Trends in Multiparameter Sensor Systems», Proceedings of the 11th European Conference on Solid State Transducers, EUROSENSORS XI, pp.527-542, 1997.

4. C.E.H.Berger, T.A.M.Beumer, R.P.H.Kooyman, J.Greve «Surface Plasmon Resonance Multisensing», Anal.Chem., 70, pp.703-706, 1998.

5. D.Farin, S.Peleg, D.Yavin, D.Avnir «Application and Limitation of Boundary-Line Fractal Analysis of Irregular Surfaces: Proteins, Aggregates, and Porous Materials», Langmuir, 1, pp.399-407, 1985.

6. F. Vogtle, Supramolecular Chemistry, London: John Wiley \& Sons., 1993.

7. J.Ramsden «Optical Biosensors», J.of Molecular Recognition , 10, pp.109-120, 1997.

8. B.A.Snopok, K.V.Kostyukevych, O.V.Rengevych, Y.M.Shirshov, E.F.Venger, I.N.Kolesnikova, E.V.Lugovskoi, «A Biosensor Approach to Probe the Structure and Function of the Adsorbed Proteins: Fibrinogen at the Gold Surface», Semiconductor Physics, Quantum Electronics \& Optoelectronics, 1, pp.121-134, 1998.

9. H.Ron, S.Matlis, I.Rubinstein «Self-Assembled Monolayers on Oxidized Metals. 2. Gold Surface Oxidative Pretreatment, Monolayer Properties, and Depression Formation», Langmuir, 14, pp.1116-1121, 1998.
10. P.Mulvaney «Surface Plasmon Spectroscopy of Nanosized Metal Particles», Langmuir, 12, pp.788-800, 1996.

11. C.Ruppe, A.Duparre, «Roughness analysis of optical films and substrates by atomic force microscopy», Thin Solid Films 288, pp.813, 1996.

12. J. M. Bennett and L. Mattsson, «Introduction to Surface Roughness and Scattering», Optical Society of America, Washington, D. C., 1989.

13. J.C.Stover, Optical scattering: measurement and analysis, McGrawHill, Inc.(1990).

14. A.S.Toporez, «Optics of rough surfaces», Mashinostroenie, Leningrad (1988).

15. H. Raether, «Surface Plasmons on Smooth and Rough Surfaces and on Gratings», Springer-Verlag, 1988.

16. A. Duparre «Light Scattering of Thin Dielectric Films» in Handbook of Optical Properties, V.1, Ed. R.E.Hummel and K.H.Guenther, CRC Press, 1995.

17. The Fractal Approach to Heterogeneous Chemistry. Ed.by D.Avnir. J.Wiley\&Sons, 1989.

18. Feder, J. Fractals (NY: Plenum Press, 1988).

19. Avnir, D., Pfeifer, P. J.Chem.Phys., 1983, 79, 3558.

20. Fractals in Physics, ed.by L.Pietronero, E.Tosatti (Elsevier Sci,Ltd, Oxford, 1986)

21. B.B.Mandelbrot, The Fractal Geometry of Nature (Freeman, San Francisco, 1983).

22. A.Bunde, S.Havlin (eds.) Fractals and Disordered Systems (Springer, 1996).

23. W.M.Tong, R.S.Williams Annu.Rev.Phys., 1994, 45, 401.

24. T.C.Halsey, M.H.Jensen, L.P.Kadanoff, I.Procaccia, B.I.Shraiman Phys.Rev.A, 1986, 22, 1141.

25. Grassberger, J. Phys.Lett.A, 1985, 107, 101.

26. J.M.Elson, «Theory of light scattering from a rough surface with an anhomogeneous dielectric permittivity», Physical Review B30, pp.5460-5480, 1984.

27. D. S. Karpovich, G. J. Blanchard, «Direct Measurement of the Adsorption Kinetics of Alkanethiolate Self-Assembled Manolayers on a Microcrystalline Gold Surface», Langmuir 10, pp.3315-3322, 1994.

28. M.-T. Lee, C.-C. Hsueh, M. S. Freund, G. S. Ferguson, «Air Oxidation of Self-Assembled Monolayers on Polycrystalline Gold: the Role of the Gold Substrate», Langmuir 14, pp.6419-6423, 1998.

29. A. G.Frutos and R. M. Corn, «SPR of ultrathin organic films», Anal. Chem. News \& Features 70, pp. 449A-455A, 1998.

30. J.A. J.A.Sanchez-Gil, J.V.Garcia-Ramos «Strong surface field enhancement in the scattering of p-polarized light from fractal metal surfaces», Optics Communications, 134, pp.11-15, 1997. 